Artigo Original

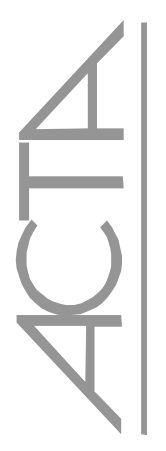

\title{
Conhecimentos de adolescentes sobre Doenças Sexualmente Transmissíveis: subsídios para prevenção
}

\author{
Teenagers' knowledge of Sexually Transmitted Diseases: strategies for prevention \\ Conocimientos de adolescentes sobre Enfermedades Sexualmente Transmisibles: \\ subsidios para la prevención
}

\section{José Roberto da Silva Brêtas ${ }^{1}$, Conceição Vieira da Silva Ohara ${ }^{2}$, Dulcilene Pereira Jardim ${ }^{3}$, Renata de Lima Muroya ${ }^{3}$}

\section{RESUMO}

Objetivo: Identificar o conhecimento de adolescentes sobre as formas de transmissão e prevenção das Doenças Sexualmente Transmissíveis. Metodos: envolveu 920 adolescentes entre 10 e 19 anos de idade eos dados foram obtidos por meio de um questionário estruturado. Resultado: os resultados demonstraram que a principal fonte para obtenção de informações sobre o assunto foi o professor; as Doenças Sexualmente Transmissíveis não são totalmente desconhecidas para os adolescentes do estudo, sendo a AIDS a mais conhecida. Descritores: Adolescente; Doenças sexualmente transmissíveis; Saúde do adolescente; Sexualidade; Vulnerabilidade em saúde.

\begin{abstract}
Objective: To identify teenager's knowledge of transmission and prevention of sexually transmitted diseases (STD). Methods: A sample of children and teenagers aged between 10 and 19 years participated in the study. Data were collected through a structured questionnaire developed for the study. Results: The findings suggested that the main source of information on Sexually Transmitted Diseases was the participants' school teacher. Sexually transmitted diseases are not entirely unknown by the participants in the study, with AIDS the most notorious.

Keywords: Teenager; Sexually transmitted diseases; Teenager health; Sexuality; Health vulnerability.
\end{abstract}

\section{RESUMEN}

Objetivo: Identificar el nivel de conocimiento de adolescentes sobre las formas de transmisión y prevención de las EST. Métodos: Envolvió 920 adolescentes entre 10 y 19 años de edad, los datos fueron obtenidos por medio de un cuestionario estructurado. Resultados: Los resultados demostraron que la principal fuente para obtención de informaciones sobre el asunto fue el profesor; las Enfermedades Sexualmente Transmisibles no son totalmente desconocidas para los adolescentes del estudio, siendo el SIDA la más conocida. Descriptores: Adolescente; Enfermedades de transmisión sexual; Salud del adolescente; Sexualidad; Vulnerabillidad en salud.

\footnotetext{
${ }^{1}$ Psicólogo, Enfermeiro, Professor Adjunto da Universidade Federal de São Paulo - UNIFESP - São Paulo (SP), Brasil.

${ }^{2}$ Enfermeira, Professora Associado da Universidade Federal de São Paulo - UNIFESP - São Paulo (SP), Brasil.

${ }^{3}$ Pós-graduanda (Mestrado em Ciências pela Universidade Federal de São Paulo - UNIFESP - São Paulo (SP), Brasil.
} 


\section{INTRODUÇÃO}

O desenvolvimento da sexualidade na adolescência tem sido tema de muitos estudos na atualidade devido a vulnerabilidades inerentes ao seu exercício neste grupo. Segundo dados da Organização Mundial da Saúde, a grande maioria dos adolescentes inicia a vida sexual cada vez mais cedo, a maioria entre 12 e 17 anos $^{(1)}$. Neste contexto, os jovens que estão vivenciando esta fase caracterizam-se, também, por sua vulnerabilidade às Doenças Sexualmente Transmissíveis (DST) e ao Vírus da Imunodeficiência Humana (HIV), e isso ocorre devido à liberação sexual, facilidade dos contatos íntimos precoces, estímulos vindos dos meios de comunicação, bem como a falta de acesso à informação e discussão sobre temas ligados a sexualidade e anticoncepção.

Assim, para este estudo adotamos a compreensão de vulnerabilidade como um movimento de considerar a chance de exposição das pessoas ao adoecimento, como resultante de um conjunto de aspectos não apenas individuais, mas também coletivos e contextuais, que acarretam maior suscetibilidade à infecção e ao adoecimento e, de modo inseparável, maior ou menor disponibilidade de recursos de todas as ordens para se proteger de ambos (2). A operacionalização se dá por meio das diferentes situações de vulnerabilidade dos sujeitos individuais e coletivos que podem ser particularizadas pela avaliação articulada de três componentes interligados: o individual (vulnerabilidade individual), o social (vulnerabilidade social) e o programático ou institucional (vulnerabilidade programática).

O componente individual da vulnerabilidade refere-se ao grau e à qualidade da informação que os indivíduos dispõem sobre o problema; à capacidade de elaborar essas informações e incorporá-las aos seus repertórios cotidianos de preocupações; e, finalmente, ao interesse, às possibilidades efetivas de transformar essas preocupações em práticas protegidas e protetoras ${ }^{(2-3)}$. Neste contexto podem ser incluídos os aspectos cognitivos, ou seja, a capacidade do indivíduo em processar informações, por exemplo, sobre DST/Aids, sexualidade, prevenção e os aspectos comportamentais que dizem respeito à possibilidade de transformar informações processadas em comportamentos.

No contexto de vulnerabilidade, a Organização Mundial da Saúde divulgou um relatório sobre a juventude, no qual consta que os jovens já representam $18 \%$ da população mundial e estão em risco aumentado, sobretudo pela vulnerabilidade à epidemia da HIV/Aids. O documento mostra que 10 milhões de jovens entre 15 a 24 anos estão infectados pelo HIV. Além disso, as doenças infecciosas como a Aids seriam responsáveis por um número duas vezes maior de mortes em 2005 quando comparadas com 2004 $4^{(4)}$.

A rápida expansão do HIV/Aids na população juvenil, especialmente entre as mulheres jovens heterossexuais, não somente obedece à falta de informação, como é o resultado de fatores culturais que operam contra a sua prevenção, fato que determina que nos países da região de menor desenvolvimento relativo é manifestado um aumento na propagação das doenças de transmissão sexual entre a população mais jovem. A incidência do HIV/Aids na mortalidade dos jovens latino-americanos de ambos os sexos (2,9 por cada 100 mil), embora inferior à dos adultos entre 25 a 44 anos (16,9 por 100 mil), não deixa de ser alarmante ${ }^{(4)}$.

As mulheres jovens têm os maiores índices da epidemia, e para elas as DST representam um sério impacto na sua saúde reprodutiva, podendo causar esterilidade, doença inflamatória pélvica, câncer de colo de útero entre outras, além de interferir negativamente sobre a auto-estima ${ }^{(5)}$.

De acordo com o DataSus, em 2007 foram registrados 13.071 casos de AIDS no Brasil, sendo 3.057 só no Estado de São Paulo. Na faixa etária de 10 a 19 anos registrou-se 269 casos no Brasil e 43 só no Estado de São Paulo; e, ainda, 12 casos no município de Embu na Grande São Paulo, e 10 casos de sífilis, sendo este município local de estudos do presente projeto( ${ }^{(6)}$.

Diante deste quadro, torna-se óbvia a necessidade do desenvolvimento de medidas preventivas para este grupo, com enfoque na orientação sexual que propicie condições para o adolescente se proteger, entre outros riscos, da transmissão das DST.

A motivação para a realização deste estudo teve como ponto de partida nossa experiência no Projeto de Extensão Universitária "Corporalidade e Promoção da Saúde", no qual desenvolvemos atividades de orientação sexual para uma população de adolescentes e jovens que freqüentam três escolas públicas da região de Santo Eduardo do município de Embu, SP. As atividades do projeto estão vinculadas ao Programa de Integração Docente-Assistencial do Embu (PIDA/EMBU), instituído desde 1970 e caracterizado pela integração de ações desenvolvidas por diferentes departamentos acadêmicos da Universidade Federal de São Paulo, com a população moradora desse município.

Para atender às necessidades do projeto, o conceito de ação adotado foi o de orientação sexual, que pode ser conceituado como o processo de intervenção sistemática na área da sexualidade humana, e que se propõe a fornecer informações sobre sexualidade e a organizar um espaço de reflexões e questionamento sobre a importância da prevenção, mudanças corporais, identidade, relações interpessoais, auto-estima, relações de gênero, tabus, crenças e valores a respeito de relacionamentos, comportamentos sexuais e DST ${ }^{(7)}$. Assim, este estudo serviu para a obtenção de dados, visando à contribuição para o conteúdo dessas atividades educacionais, norteando nosso fazer e buscando atender ás reais necessidades da população envolvida.

Neste contexto, o presente estudo teve como objetivo identificar o nível de conhecimento dos adolescentes sobre as formas de transmissão e prevenção das DST.

\section{MÉTODOS}

Trata-se de um estudo descritivo que visa promover o delineamento das características da população, de fatos e 
fenômenos envolvidos com a realidade. O mesmo proporciona o conhecimento da realidade, uma vez que descreve, registra, analisa e interpreta a natureza atual ou processos dos fenômenos ${ }^{(8)}$.

O projeto deste estudo foi avaliado pelo Comitê de Ética em Pesquisa da Universidade Federal de São Paulo (UNIFESP), Protocolo n ${ }^{\circ} 1561 / 03$, obedecendo todo padrão estabelecido pela Resolução 196/96, que trata das Normas de Pesquisa Envolvendo Seres Humanos ${ }^{(9)}$.

Este estudo foi realizado após assinatura do Termo de Consentimento Livre e Esclarecido, no período entre 2004 e 2005 , entre 920 adolescentes de três escolas públicas de ensino fundamental e médio na região de Santo Eduardo da Estância Turística de Embu, São Paulo. Nestas escolas, onde são desenvolvidas as atividades de educação para saúde do Projeto de Extensão Universitária "Corporalidade e Promoção da Saúde" da UNIFESP.

Como princípio de inclusão adotou-se: estar matriculado em uma das três escolas e participar das atividades educativas do Projeto de Extensão Universitária "Corporalidade e Promoção da Saúde".

A coleta de dados ocorreu mediante a aplicação de um questionário semi-estruturado, de múltipla escolha, autoaplicado em sala de aula, e de forma anônima, composto por 17 questões representando as variáveis relacionadas aos dados sócio-demográficos (idade, sexo, escolaridade, religião, estado civil) para caracterização da população estudada e as variáveis sobre o conhecimento das formas de transmissão e prevenção das DST.

Os dados obtidos foram analisados e interpretados em um contexto quantitativo, expressos mediante símbolos numéricos.

\section{RESULTADOS}

Da população constituída por 920 adolescentes, $52 \%$ eram do sexo feminino, na faixa etária entre 10 e 19 anos, com maior concentração (68\%) entre 10 e 14 anos de idade. Encontravam-se cursando o Ensino Fundamental 81\% deles, enquanto $16 \%$ o Ensino Médio e 3\% não responderam. Com relação ao estado civil, $83 \%$ eram solteiros, apenas $1 \%$ declarara ser casado e $16 \%$ não responderam. Quanto à religião, $55 \%$ referiram ser católicos, seguidos dos evangélicos $(17 \%)$, espíritas $(1 \%)$, budistas (1\%), testemunha de Jeová (1\%), os agnósticos $(15 \%)$ e $10 \%$ não responderam à questão.

Os resultados relacionados ao conhecimento dos adolescentes sobre as formas de transmissão e prevenção das DST estão apresentados nos Quadros 1 e 2.

Além dos sinais e sintomas da AIDS descritos no Quadro 2 , ainda foram mencionados outros como: cansaço (32\%), falta de apetite $(30 \%)$, dor no corpo e articulações $(31 \%)$, febre (31\%), suores noturnos (21\%), "sapinhos" na boca ( $8 \%$ ), pingos hemorrágicos que aparecem na pele $(15 \%)$, tosse persistente $(15 \%)$ e diarréia (11\%).

Quanto à possibilidade de cura das DST/AIDS, os resultados apontaram que os adolescentes relacionavam a cura para gonorréia (25\%), herpes genital (17\%), Sífilis (18\%), Condiloma acuminado (9\%), Aids (9\%), $12 \%$ afirmaram que nenhuma das DST/Aids tem cura, e $48 \%$ não souberam responder.

Com relação à prevenção, os adolescentes foram argüidos sobre as ações realizadas para prevenir as DST/Aids, sendo que esta questão admitiu mais de uma resposta. Os resultados apontaram que $89 \%$ usavam o condom em todas as relações sexuais como forma de prevenção, $72 \%$ afirmaram

Quadro 1 - População estudada segundo o conhecimento sobre as formas de contágio das DST. Embu, São Paulo, Brasil, 2004-2005\%.

\begin{tabular}{|c|c|c|c|c|c|}
\hline \multirow[b]{2}{*}{ Vias de Contágio } & \multicolumn{5}{|c|}{ DST } \\
\hline & $\begin{array}{c}\text { Sífilis } \\
\text { n }\end{array}$ & $\underset{\mathbf{n}}{\text { Gonorréia }}$ & $\begin{array}{l}\text { Herpes Genital } \\
\mathbf{n}\end{array}$ & $\begin{array}{c}\text { Condiloma } \\
n\end{array}$ & $\underset{\mathbf{n}}{\operatorname{AIDS}}$ \\
\hline Sexo vaginal & 30 & 24 & 23 & 10 & 76 \\
\hline Sexo oral & 14 & 9 & 12 & 7 & 37 \\
\hline Sexo anal & 13 & 18 & 12 & 7 & 44 \\
\hline Da mãe para o filho & 9 & - & 3 & 2 & 34 \\
\hline Contato com a ferida & 11 & - & - & - & - \\
\hline Transfusão sanguínea & 10 & - & 5 & 3 & 60 \\
\hline No beijo/saliva & 4 & 1 & 3 & 3 & 3 \\
\hline No abraço/mãos & 1 & 1 & 1 & - & 8 \\
\hline $\begin{array}{l}\text { Sentar no mesmo lugar/vaso } \\
\text { sanitário }\end{array}$ & 1 & 7 & - & 3 & 15 \\
\hline Roupas íntimas & - & 5 & - & - & - \\
\hline Drogas injetáveis & - & - & - & - & 55 \\
\hline Não sei & 64 & 66 & 68 & 87 & 9 \\
\hline Não responderam & 3 & 1 & 1 & 1 & 1 \\
\hline
\end{tabular}

(*) Admitiu-se assinalar mais de uma resposta. 
Quadro 2 - População estudada segundo o conhecimento sobre os sinais e sintomas das DST. Embu, São Paulo, Brasil, 2004-2005*.

\begin{tabular}{|c|c|c|c|c|c|}
\hline \multirow[b]{2}{*}{ Sinais e sintomas } & \multicolumn{5}{|c|}{ DST } \\
\hline & $\begin{array}{c}\text { Sífilis } \\
\mathbf{n}\end{array}$ & $\begin{array}{c}\text { Gonorréia } \\
\text { n }\end{array}$ & $\begin{array}{c}\text { Herpes Genital } \\
\text { n }\end{array}$ & $\begin{array}{c}\text { Condiloma } \\
\mathbf{n}\end{array}$ & $\begin{array}{c}\text { AIDS } \\
\text { n }\end{array}$ \\
\hline Feridas nos órgãos genitais & 32 & 3 & 8 & 6 & - \\
\hline Manchas pelo corpo & 7 & - & - & - & - \\
\hline Coceira & 8 & 4 & 3 & 4 & 19 \\
\hline Corrimento & 6 & 20 & 3 & - & 13 \\
\hline Odor forte da secreção & 7 & - & - & 6 & - \\
\hline Perda de cabelos & 1 & 1 & 1 & 1 & - \\
\hline Morte & 2 & - & - & - & - \\
\hline Impotência & 1 & 1 & 1 & 1 & 4 \\
\hline Dor ao evacuar & - & 5 & - & - & - \\
\hline Faringite & - & 1 & - & - & - \\
\hline Saída sangue/secreção purulenta & - & 4 & 8 & 3 & - \\
\hline Ínguas & - & - & 2 & - & 10 \\
\hline Dor/dificuldade para urinar & - & 11 & 5 & 4 & - \\
\hline Dor local & 7 & - & 12 & 4 & - \\
\hline Não sei & 70 & 73 & 85 & 89 & 47 \\
\hline Não responderam & 1 & 2 & 2 & 2 & 1 \\
\hline
\end{tabular}

que consultar um médico regularmente era uma medida preventiva, $68 \%$ certificavam-se de que o parceiro sexual não possuía nenhuma DST, 45\% lavavam os genitais após a relação sexual, apenas $6 \%$ não indicaram nenhuma medida.

Como fonte de informações sobre as DST/Aids, $71 \%$ dos adolescentes consideraram os professores a principal fonte de informações e a televisão como segunda opção (55\%). Seguiram-se as informações obtidas por meio de jornais e revistas (47\%), a família e os amigos (36\%) igualmente classificados, e 3\% não obtinham nenhum tipo de informação.

\section{DISCUSSÃO}

As vias de contágio das DST mais citadas foram as sexuais (sexo vaginal, anal e oral respectivamente), possivelmente por serem estes os meios mais comuns de contágio das doenças, e também os mais difundidos pelos meios de comunicação e em campanhas voltadas para a prevenção. Este resultado corrobora outros estudos.

Os indivíduos constroem suas próprias definições de risco de transmissão do HIV, por meio da via sexual, utilizando parte do discurso epidemiológico e preventivo, mas principalmente de acordo com sua posição social, identidade pessoal e tipo de relação na qual estão envolvidos ${ }^{(10)}$.

A realidade, porém, mostra que grandes proporções de adolescentes se engajam em contatos sexuais, como sexo oral e anal, sem reconhecê-los como fonte de contágio de DST. O contágio das DST por uso do banheiro, pela saliva, roupas, de mãe para filho e via sanguínea também foram apontados em outros estudos ${ }^{(10)}$.

A DST mais conhecida é a Aids, enquanto o Condiloma
Acuminado (Papiloma Vírus Humano/HPV) mostrou-se o menos conhecido, confirmando outro estudo realizado com adolescentes na periferia da cidade de São Paulo ${ }^{(11)}$. Trata-se de um dado preocupante, pois a adolescência é a faixa de idade que apresenta a maior incidência de DST. Aproximadamente $25 \%$ de todas as DST são diagnosticadas em jovens com menos de 25 anos. Os dados disponíveis de âmbito mundial ${ }^{(12)}$ revelaram que aproximadamente $40 \%$ das adolescentes sexualmente ativas foram infectadas pelo Papilomavírus humano(HPV), responsável pelo Condiloma Acuminado que é uma doença infecciosa, conhecida como verruga genital ou popularmente chamada de crista de galo ${ }^{(13)}$. Não menos preocupante, a infecção pelo vírus do Herpes Genital (Herpes simplex vírus) aumentou em mais de 50\% nesta população(12).

O estudo mostra que uma grande porcentagem dos adolescentes não conhecia nenhuma forma de contágio das DST, o que nos mostra que, apesar da difusão pelos veículos sociais de informação, esta não tem alcançado a população de forma efetiva.

Quanto aos sinais e sintomas das DST, o aparecimento de feridas nos órgãos genitais foi o mais apontado, seguido do corrimento e coceira nestes órgãos. A impotência e a coceira foram associadas a todas as DST. Da mesma forma, houve um conhecimento mais expressivo em relação aos sinais e sintomas da Aids em relação às demais DST.

As porcentagens de adolescentes que desconheciam os sinais e sintomas das DST foram ainda maiores em relação ao desconhecimento das formas de contágio.

Quanto à possibilidade de cura, a gonorréia teve a maior porcentagem, seguida da Sífilis e Herpes Genital, tendo a Aids e o Condiloma Acuminado a mesma representação 
numérica. Uma parcela significativa dos adolescentes não atribuiu cura a nenhuma das DST, e quase a metade da população não soube responder a questão.

As DST são hoje consideradas um grave problema de saúde pública por sua magnitude, pela dificuldade das pessoas identificarem seus sintomas e, principalmente, por serem grandes facilitadores da transmissão do HIV. O tratamento imediato das DST é, portanto, de importância fundamental, pois as feridas, inflamações, corrimentos e verrugas nos órgãos genitais são portas de entrada para outras DST.

Com exceção das causadas por vírus (Aids, HPV e Herpes), existe cura para todas DST, se o tratamento for feito adequadamente. $\mathrm{Na}$ maioria das vezes, este é feito à base de antibióticos. Deve-se salientar que um tratamento eficiente envolve cuidados médicos, higiene pessoal, medicação adequada e aconselhamento. Envolve, também, o tratamento rápido e breve das parcerias sexuais, postura que reflete a preocupação com a interrupção da cadeia de transmissão das DST.

Quanto às fontes de informação sobre as DST, o professor foi apontado como primeira opção entre os adolescentes, confirmando a sua importância na função natural de educador sexual no ambiente escolar. Este fato que reforça a tese de que é necessária a atualização contínua do conhecimento desses profissionais sobre sexualidade e DST, para cumprimento eficaz do seu papel.

Este resultado confirma a importância da escola como cenário privilegiado de acolhimento contínuo de adolescentes e jovens, e enquanto espaço para o compartilhamento de decisões e responsabilidades com as demais instâncias sociais envolvidas na efetivação das estratégias articuladas de redução da vulnerabilidade de adolescentes.

Neste sentido, o ambiente escolar é um espaço institucional privilegiado para convivência social e o estabelecimento de relações intersubjetivas favoráveis à promoção da saúde e à construção de resposta social aos desafios colocados para a sociedade. $\mathrm{Na}$ construção de espaços de diálogo entre adolescentes, jovens, professores, profissionais de saúde e comunidade é, comprovadamente, um importante dispositivo para construir resposta social com vistas à superação das relações de vulnerabilidade às DST, à infecção por HIV e à Aids, assim como à gravidez não planejada. Para tanto, as ações desenvolvidas devem ir além da dimensão cognitiva, levando em conta aspectos subjetivos, questões relativas às identidades e às práticas afetivas e sexuais no contexto das relações humanas e dos direitos humanos ${ }^{(14)}$.

Em um estudo realizado com 100 professores da rede pública no município de Jandira, São Paulo, apenas 30\% achavam que as DST deveriam fazer parte do currículo de educação sexual na escola, sendo que $14 \%$ consideravam difícil falar sobre esse assunto, e $4 \%$ sentiam-se totalmente incapacitados para a função ${ }^{(15)}$. Estes resultados demostraram a necessidade de treinamentos específicos sobre o tema sexualidade para professores, e constante reciclagem de conhecimentos para que estes desenvolvam, com segurança, as atividades de orientação sexual no âmbito escolar.

As demais fontes de informação apontadas pelos adolescentes são os meios de comunicação de massa, os amigos e a família, dado confirmado por outros estudos ${ }^{(16)}$. A educação sexual é prioritariamente uma função da família, mas muitas vezes os pais têm dificuldades de abordar questões relacionadas ao assunto sexualidade com os filhos adolescentes, justamente por não terem muito claro o que aconteceu com eles próprios. Desta maneira, muitos pais atribuem a tarefa da orientação sexual de seus filhos à escola e esta, por sua vez, apresenta dificuldade em cumprir tal tarefa ${ }^{(7)}$.

As informações sobre formas de transmissão e prevenção, por si só, não são suficientes para a adoção de comportamentos protetores. Porém, a falta de informações básicas contribui para aumentar a vulnerabilidade dos adolescentes.

Quanto às formas de prevenção das DST, de suma importância para este estudo, o uso da camisinha em todas as relações sexuais foi apontado como a primeira escolha de prevenção, o que demonstra um bom nível de conhecimento preventivo por parte dos adolescentes, e que coincide com outros estudos ${ }^{(17)}$.

No Brasil, assim como em outros países, tem havido um significante aumento do uso da camisinha pelos adolescentes $^{(18)}$, porém esta ainda não é usada por todos e nem em todas as relações sexuais. Restam ainda adolescentes que referem nunca ter usado o preservativo, apesar de conhecerem os riscos aos quais estavam expostos ${ }^{(19)}$. O seu uso depende, entre outros fatores, do envolvimento afetivo do momento, questões financeiras e de acesso aos métodos, bem como o grau de liberdade e autonomia alcançadas nesta faixa etária ${ }^{(18)}$.

Uma pesquisa nacional, realizada em 2004, indicou que $57,3 \%$ dos jovens entre 15 e 24 anos usaram preservativos na última relação sexual, $58,5 \%$ sempre usaram preservativo com parceiro casual, e $38,8 \%$ usaram esse método com parceiro fixo ${ }^{(18)}$.

Ainda como forma de prevenção, grande porcentagem de adolescentes apontou que consultar o médico regularmente pode prevenir as DST. Certificar-se de que o parceiro não possua nenhuma doença também foi citada em outros estudos $^{(11)}$. O fato de pensar conhecer o (a) parceiro (a) elimina, em nível imaginário, todos os riscos de se adquirir uma DST/Aids, o que coloca em risco o adolescente, pois as DST têm um período de latência para o aparecimento de sintomas, além de formas subclínicas, não perceptíveis ao contato sexual.

A adoção da prática de lavar os órgãos genitais após a relação sexual como medida preventiva revela a imaturidade do pensamento adolescente e seu comportamento de onipotência.

A onipotência constitui uma característica importante e freqüente entre adolescentes. Este exercício de autoafirmação faz com que pensem ter imunidade contra tudo que acontece ou possa acontecer em seu meio, acham que 
nada de mal irá acontecer consigo. O pensamento do adolescente frente ao tempo também expressa uma defesa muito importante, uma vez que a busca da identidade adulta está intimamente ligada a capacidade de conceituar o tempo; nesta fase de transição, vive intensamente o presente, não aceitando o futuro ${ }^{(20)}$.

Concluindo a questão, uma pequena, mas importante parcela de adolescentes acredita que nunca contrairá nenhum tipo de DST, expressando mais uma vez o senso de invulnerabilidade próprio da adolescência. Talveza resposta para este grupo esteja nas próprias características da fase de adolescer, ou seja, sentimento de onipotência, que o faz sentirse imune aos perigos, desafiar regras, crer que esteja isento das conseqüências dos perigos a que se expõem ${ }^{(21)}$.

Um estudo realizado com adolescentes da rede pública e privada em São Paulo demonstrou que a maior parte desta população possuía conhecimento adequado sobre a transmissão e prevenção das $\operatorname{DST}^{(18)}$.

De um modo geral, os adolescentes possuem maior conhecimento sobre as DST que os adultos, e o nível de informação aumenta conforme ocorre a melhoria nas condições sócio-econômicas, somando-se, ainda a questão de gênero, em que as mulheres se mostram mais bem informadas do que os homens ${ }^{(10,17)}$. No entanto, embora exista a informação, essa compreensão é escassa e insuficiente para promover um comportamento sexual seguro(18).

As condições que afetam a vulnerabilidade individual são de ordem cognitiva - informação, consciência do problema e das formas de enfrentá-lo, comportamentais - interesse e habilidade para transformar atitudes e comportamento a partir daqueles elementos cognitivos, e sociais - acesso a recursos e poder para adotar comportamentos protetores.

\section{CONCLUSÃO}

Os subsídios para prevenção proporcionados por este estudo mostraram a dificuldade que a grande maioria dos adolescentes tem em relação ao conhecimento das formas de contágio das DST, embora demonstrem que a Aids é mais conhecida. Esse fato se deve, especialmente, à informação sobre prevenção da Aids pela mídia, o que mostra que o investimento em informação nesse veículo de comunicação

\section{REFERÊNCIAS}

1. Castro MG, Abramovay M, Silva LB. Juventudes e sexualidade. Brasília: UNESCO; 2004.

2. Ayres JRCM, França Junior I, Calazans GJ, Filho HCS. O conceito de vulnerabilidade e as práticas de Saúde: novas perspectivas e desafios. In: Czeresnia D, Freitas CM. Promoção da saúde: conceitos, reflexões, tendências. Rio de Janeiro: Fiocruz; 2003. p.117-39.

3. Muñoz Sánchez AI, Bertolozzi MR. Pode o conceito de vulnerabilidade apoiar a construção do conhecimento em Saúde Coletiva? Ciênc Saúde Coletiva. 2007; 12(2):319-24.

4. Plano Ibero-Americano de Cooperação e Integração da de massa é um dos caminhos para a prevenção que pode gerar mudanças no comportamento dos indivíduos.

O estudo também demonstrou grande desconhecimento sobre os sinais e sintomas das DST, inclusive da Aids. Embora a maioria dos adolescentes pesquisados tenha referido utilizar o condom como forma de prevenção, um numero expressivo (45\%) acreditava que apenas a higiene após o ato sexual pode se constituir em um meio de prevenção.

Estas informações serviram e servem para nortear as nossas ações educativas e reforçar nossa convicção sobre a importância da orientação sexual na escola, e a conduta de pesquisar e intervir, enquanto metodologia para atender a ampliação dos canais de percepção e comunicação, para possibilitar a compreensão das distintas realidades com as quais entramos em contato.

A partir deste contexto, procuramos abordar a sexualidade como um aspecto natural e positivo da vida humana, proporcionando a livre discussão de normas e padrões de comportamento em relação ao sexo e o debate das atitudes das pessoas frente à própria sexualidade, enfatizando aspectos específicos sobre as DST, como as formas de contágio, os sinais e sintomas e as formas de prevenção.

No sentido de acolher este adolescente, ajudá-lo a se apropriar do conhecimento, promover mudanças em seu comportamento, encontramos no ambiente escolar o campo adequado para esta finalidade.

Vale ressaltar a importância do professor, que foi indicado como principal fonte de informação por este estudo, e que precisa de apoio e ajuda para reciclar seus conhecimentos técnicos e, ainda, para superar as questões relacionadas à sua própria educação sexual. Assim, como forma de capacitar e instrumentalizar os professores, elaboramos um curso de extensão junto às escolas que fazem parte do campo de ação do Projeto de Extensão Universitária Corporalidade e Promoção da Saúde.

Desta forma, as pesquisas subsidiam as ações para vencer as barreiras, ajudando a mostrar que, para ensinar adolescentes, é preciso transformar o conhecimento em caso pessoal, praticar a subjetivação do conhecimento, ou seja, transformar espectadores, nem sempre muito interessados, em atores que entendam e direcionem, de forma consciente, a sua história sexual-afetiva.
Juventude. 2009-2015 [Internet]. [citado 2008 Out 12]. Disponível em: http://www.oij.org/documentos/ doc1226582177.pdf

5. Dollabetta G, Lyn M, Laga M, Islam M. DST: impacto global do problema e desafios para o controle. In: Dollabetta G, Laga M, Lamptey P. Controle de doenças sexualmente transmissíveis: manual de planejamento e coordenação de programas. Rio de Janeiro: Te Cora Editora/Associação Saúde da Família;1997. p. 1-22.

6. Ministério da Saúde (BR). DataSus [Banco de dados da Internet]. [Acesso em 2009 março 20]. Disponível em: http://www.datasus.gov.br 
7. Brêtas JRS, Pereira SR. Projeto de extensão universitária: um espaço para formação profissional e promoção da saúde. Trab Educ Saúde. 2007;5(2):317-27.

8. Gil AC. Métodos e técnicas de pesquisa social. São Paulo: Atlas; 2003.

9. Ministério da Saúde (BR). Conselho Nacional de Saúde. Resolução n.196 de 10 de outubro de 1996: Diretrizes e normas regulamentadoras de pesquisas em seres humanos. Mundo Saúde. 1996;21(1):52-61.

10. Ferreira MP. Conhecimento e percepção de risco sobre o HIV/AIDS: um perfil da população brasileira no ano de 1998. Cad Saúde Pública. 2003;19(Supl 2):S213-22.

11. Silva CV, Brêtas JRS, Fernandes CN. Conhecimento de adolescentes sobre doenças sexualmente transmissíveis/ AIDS. Rev Paul Enferm. 2003;22(1):12-21.

12. Kaplan DW, Feinstein RS, Fisher MM, Klein JD, Olmedo LF, Rome ES, et al. Committee on Adolescence. Condom use by adolescents. Pediatrics. 2001; 107(6):1463-9.

13. Brêtas JRS, Oliveira JR, Aguiar Junior W. Doenças sexualmente transmissíveis. In: Brêtas ACP, Gambá MA. Enfermagem e saúde do adulto. São Paulo: Manole; 2006. p. 249-63.

14. Ministério da Saúde (BR). Secretaria de Vigilância em Saúde. Programa Nacional de DST e Aids. Diretrizes para implantação do Projeto Saúde e Prevenção nas Escolas. Brasília: Ministério da Saúde; 2008.
15. Jardim DP, Brêtas JRS. Orientação sexual na escola: a concepção dos professores de Jandira - SP. Rev Bras Enferm. 2006;59(2):157-62.

16. Francisco MTR, Oliveira DC, Clos AC, Santos NC, Malaquias JV. O carnaval vai contagiar: DST/AIDS e práticas sexuais no Rio de Janeiro. Rev Enferm UERJ. 2004;12(1):30-7.

17. Teixeira AMFB, Knauth DR, Fachel JMG, Leal AF. Adolescentes e uso de preservativos: as escolhas dos jovens de três capitais brasileiras na iniciação e na última relação sexual. Cad Saúde Pública. 2006;22(7):1385-96.

18. Martins LBM, Costa-Paiva LHS, Osis MJD, Sousa MH, Pinto-Neto AM, Tadini V. Fatores associados ao uso de preservativo masculino e ao conhecimento sobre DST/ AIDS em adolescentes de escolas públicas e privadas do Município de São Paulo, Brasil. Cad Saúde Pública. 2006;22(2):315-23.

19. Szwarcwald CL, Barbosa-Júnior A, Pascom AR, SouzaJúnior PR. Knowledge, practices and behaviours related to HIV transmission among the Brazilian population in the 14-54 years age group, 2004. AIDS. 2005;19 Suppl 4:S51-8.

20. Aberastury A, Knobel M. Adolescência normal. Porto Alegre: Artes Médicas; 1981.

21. Silva CV, Brêtas JRS, Ferreira D, Correa DS, Cintra CC. Uso da camisinha por adolescentes e jovens: avaliação da seqüência dos procedimentos. Acta Paul Enferm. 2004;17(4):392-9. 